\title{
Fair Power Allocation in Cooperative Cognitive Systems under NOMA Transmission for Future IoT Networks
}

\author{
Zain Ali, Wali Ullah Khan, Guftaar Ahmad Sardar Sidhu, Nimmi K, Xingwang Li, \\ Kyung Sup Kwak, and Muhammad Bilal
}

\begin{abstract}
To support the massive connectivity in Internet of Things (IoT), several promising techniques like cognitive radio (CR) and non-orthogonal multiple access (NOMA) have come forward. These techniques enable the users to share the spectrum resources. However, such cooperation can degrade performance of helping users. This work aims to achieve fairness among secondary users (SUs) in IoT cooperative NOMA-based CR transmission. We design a power allocation algorithm for secondary IoT network that ensures protection of primary system. Further, an independent battery constraint at each node is considered, and power gap among transmissions of two NOMA users is applied for successive interference cancellation. Our extensive simulation results show that the proposed framework provides excellent performance and for sufficient available transmission power perfect fairness is achieved in the rate of both the users.
\end{abstract}

Index Terms-Cognitive radio; Cooperative communication; Internet of Things; Non-orthogonal multiple access; Power optimization

\section{INTRODUCTION}

The use of orthogonal multiple access (OMA) systems is well known because of their excellent performance [1], [2], [3]. However, the Internet of Things (IoT) demands high spectral efficiency (SE) which the OMA systems are unable to provide [4], [5]. Hence, researchers have recently been working on finding a better multiple access substitute. Nonorthogonal multiple access (NOMA) provides better performance as compared to OMA and is a potential candidate for the IoT systems [6], [7]. NOMA offers high spectral efficiency because it enables multiple IoT users to simultaneously share a single channel [8]. This can be accomplished by superimposing multiple signals at the transmitter side using the superposition coding technique and then successfully decode it at the receiver side with the help of successive interference cancellation technique (SIC) [9]. Another network that provides high spectral efficiency by employing spectrum reuse

Z. Ali and G. A. S. Sidhu are with the Department of Electrical and Computer Engineering, COMSATS University Islamabad, Park Road, Chak Shahzad, Islamabad, Pakistan (emails: zainalihanan1@gmail.com, guftaarahmad@comsats.edu.pk)

W. U. Khan is with Interdisciplinary Center for Security, Reliability and Trust (SnT)/ SigCom, University of Luxembourg, Luxembourg (email: waliullahkhan30@gmail.com)

N. K is with the Department of Computer Applications, National Institute of Technology, Trichy, India (email: nimmik4u@gmail.com)

$\mathrm{X}$. Li is with the School of Physics and Electronic Information Engineering, Henan Polytechnic University, Jiaozuo, China (email: lixingwangbupt@gmail.com)

K. S. Kwak is with the Department of Information and Communication Engineering, Inha University, Incheon, 22212, Korea. (email: kskwak@inha.ac.kr)

M. Bilal is with the Department of Computer and Electronics Systems Engineering, Hankuk University of Foreign Studies, Gyeonggi-do, 17035, Korea (email: m.bilal@ieee.org) is cognitive radio (CR). In the CR systems, the unlicensed secondary users (SRs) share the channels of the licensed primary users (PUs). However, in this system, the SUs are bound to keep the generated interference below a tolerable threshold [10].

In the advanced communication networks, optimization becomes necessary to obtain the desired performance [11], [12]. Optimal power allocation is very important to enhance the performance of the communication networks [13], [14]. Thus, in the literature the problem of power optimization for NOMA and CR based systems have been considered many times. In [15], the power allocation was optimized such that the rate demand of each user is satisfied. The authors in [16] proposed a power optimizing framework for maximizing the energy efficiency of NOMA networks. The problem of secrecy rate maximization in NOMA was studied in [17]. Then, the authors in [18] optimized power loading for rate maximization in NOMA networks. However, the results showed that the designed framework sacrifices user-fairness to increase the sum rate of the system. Hence, a sequential quadratic programming based framework was proposed in [19] to achieve fairness in the rates of all the users in a NOMA system. The results showed that the convergence rate of sequential quadratic programming is slow, thus the solution may not be useful in practical scenarios. For CR, the problem of power optimization for energy efficiency maximization was considered in [20]. In [21], power distribution was optimized to provide security in CR networks. Then, the problem of optimal power distribution for sum rate maximization was solved in [22]. In [23], the authors optimized power loading to attain fairness in CR. Similarly, several researches have also investigated optimization framework in backscatter-enabled NOMA networks [24], [25], [26].

Recently, the idea of cooperative communication has come forward as a technique that can further improve the performance of a communication networks [27]. In NOMA, a superimposed signal containing symbols of all the users is transmitted to all the receivers. A user with better channel condition (strong user) employs successive interference cancellation (SIC) to decode the intended symbol. For this, the strong user first decodes the symbols of all the users having comparatively bad channel conditions (weak users) and then remove these symbols from the received signal [28]. The strong user can then cooperate by relaying the decoded data to the weak users. This cooperation can further improve the performance of the communication system. The problem of optimizing power loading for enhancing the energy efficiency in cooperative NOMA was considered in [29]. In [30], a 
power loading technique was proposed for cooperative NOMA systems where the objective was to enhance the security of the system. For rate maximization in cooperative NOMA, the authors in [31] proposed a power allocation framework. Considering a cooperative CR system where the two SUs present in the system relay each others data to the secondary receiver, the authors in [32] worked to enhance the energy efficiency of the system. Later, the authors in [33] studied power allocation for secure communication in cooperative $\mathrm{CR}$ systems.

A NOMA based CR system can provide further improvement in the performance of the network. Hence, the combination of NOMA and CR has also been considered by some works in the literature. The authors in [34] optimized the power loading such that the number of secondary users that can access the channel is maximized. The problem of maximizing rate in NOMA has been considered in [35]. In these interference susceptible systems, the task of achieving fairness among users might become very critical for the successful operation. Further, a cooperative NOMA-CR system has the combined benefits of all the systems discussed till now. To the best of our knowledge, the problem of optimizing resource allocation to achieve fairness among the secondary IoT users in a cooperative NOMA based CR system has not been studied yet. Thus, to bridge this gap, we aim to provide an efficient solution for power allocation to achieve fairness among the IoT users in a cooperative NOMA underlay system. For this, we propose a bisection method based framework for power allocation at the transmitters, subject to the independent battery capacity of each node. The main contributions of this paper can be summarized as:

- To achieve fairness in the rates of the SU in IoT, a power allocation problem is formulated under constraints of individual power budget at the transmitter and relaying node, interference temperature, and NOMA requirement of adequate gap in the received powers for successive interference cancellation.

- We propose a cooperative transmission where we consider decode and forward relaying at the cooperative-node (strong IoT user), and the transmission is completed in two time slots. In the first slot, the transmitter (ST) sends data to the receivers. In the second slot, the strong IoT user relays the data to the far away user (weak IoT user). The received signals at the weak user in the two time slots are merged by using maximum ratio combining.

- An efficient solution based on the bisection method is provided for the optimal power allocation at the transmitting nodes. The simulation results show that the proposed framework achieves perfect fairness while providing protection to the primary system. For very small values of the available power and interference threshold, the results show that it becomes impossible to provide perfect fairness while satisfying all the constraints. However, the proposed framework provides excellent performance at these points as well.

The remaining of the paper is organized as follows: Section 2 discusses the considered system model and problem formu-

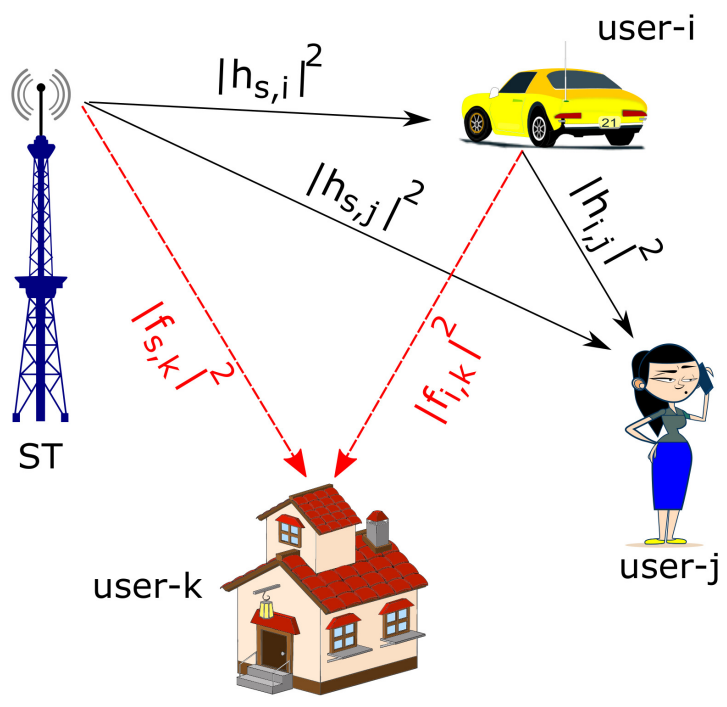

Fig. 1: Considered System Model

lation. The proposed solution is explained in section 3. The simulation results are provided in the section 4 . Finally, the work is concluded in section 5 .

\section{System Model AND PROBlem Formulation}

The considered system model is shown in Fig. 1. We have considered a NOMA based cooperative underlay IoT system containing two secondary IoT receivers ${ }^{1}$ : user $-i$ that is closer to the ST hence is also called the strong IoT user, and a $u s e r-j$, which is comparatively far from the ST, thus is the weak IoT user. We assume that all the devices in the network is equipped with omni-directional antenna and the channel state information is also available [36]. We also assume that the channels are independent and identical Raleigh fading [37], [38]. The ST transmits data to both IoT users in the presence of $u s e r-k$ which is the primary receiver (PR). The available time is split into two slots of equal duration. First, ST employs NOMA to transmit data to both the IoT users on the same channel, simultaneously. Then, in the second slot the $u s e r-i$ relays the data to the $u s e r-j$. In the system model $\left|h_{s, i}\right|^{2},\left|h_{s, j}\right|^{2}$ and $\left|h_{i, j}\right|^{2}$ represent the channel gains from ST to user- $i$, ST to the user- $j$ and from the user- $i$ to the $u s e r-j$, respectively. Further, $\left|f_{s, k}\right|^{2}$ and $\left|f_{i, k}\right|^{2}$ are the gains of interference channels from ST to user- $k$ and from $u s e r-i$ to the $u s e r-k$, respectively. In CR systems, the PR must be protected from harmful interference. In the considered framework, the available time is divided into two equal slots. Hence, we consider separate and independent interference thresholds for both time slots.

The signal received by the user- $i$ in the first time slot is given as:

$$
r_{s, i}=\left(\sqrt{P_{s, i}} z_{s, i}+\sqrt{P_{s, j}} z_{s, j}\right) h_{s, i}+n_{i}
$$

${ }^{1}$ Note that, the proposed solution can be easily extended to a large network containing multiple users. For example, in the case of a heterogeneous network, the intra-cell communication can employ NOMA transmission as considered in this paper and for the inter-cell transmission OMA can be used. 
where $n_{i}$ is the additive white Gaussian noise (AWGN) at the user-i, $P_{s, i}$ and $P_{s, j}$ are the allocated power of ST for IoT $u s e r-i$ and $u s e r-j$, respectively. Furthermore, $z_{s, i}$ and $z_{s, j}$ are the unit power symbols of IoT user- $i$ and $u s e r-j$. The data rate of IoT user-i is calculated as:

$$
R_{s, i}=\frac{1}{2} \log _{2}\left(1+\frac{P_{s, i}\left|h_{s, i}\right|^{2}}{\sigma^{2}}\right),
$$

where $\sigma^{2}$ is the variance of AWGN. Similarly, in the first time slot, the signal received by the IoT user- $j$ is:

$$
r_{s, j}=\left(\sqrt{P_{s, i}} z_{s, i}+\sqrt{P_{s, j}} z_{s, j}\right) h_{s, j}+n_{j},
$$

where $n_{j}$ is the AWGN at the ST to IoT user- $j$ link and $P_{s, j}$ is the power allocated by ST for IoT $u s e r-j$. The signal received at the user- $j$ in second time slot is:

$$
r_{i, j}=\sqrt{P_{i, j}} z_{s, j} h_{i, j}+n_{i j},
$$

where $n_{i j}$ and $P_{i, j}$ represents the AWGN at user- $i$ to $u s e r-j$ link and power invested by the user-i for relaying, respectively. We employ maximum ratio combining (MRC) to combine the signals received by the IoT user- $j$ in both time slots. Thus, the total rate of $u s e r-j$ is calculated as:

$$
R_{s, j}=\min \left\{\frac{1}{2} \log _{2}\left(1+\gamma_{s, j}+\gamma_{i, j}\right), \frac{1}{2} \log _{2}\left(1+\gamma_{s, i->j}\right)\right\},
$$

where $\gamma_{s, i->j}=\frac{P_{s, j}\left|h_{s, i}\right|^{2}}{P_{s, i}\left|h_{s, i}\right|^{2}+\sigma^{2}}, \gamma_{s, j}=\frac{P_{s, j}\left|h_{s, j}\right|^{2}}{P_{s, i}\left|h_{s, j}\right|^{2}+\sigma^{2}}$, and $\gamma_{i, j}=\frac{P_{i, j}\left|h_{i, j}\right|^{2}}{\sigma^{2}}$. Here $\gamma_{s, i->j}, \gamma_{s, j}$ and $\gamma_{i, j}$ denote the signal to interference plus noise ratio at the links between ST to IoT user- $i$, ST to IoT user- $j$ and user- $i$ to user- $j$, respectively. As $\left|h_{s, i}\right|^{2}>\left|h_{s, j}\right|^{2}$, thus it can be concluded that $\gamma_{s, i->j}>\gamma_{s, j}$. If $P_{i, j}$ is allocated such that $\gamma_{s, i->j}<$ $\gamma_{s, j}+\gamma_{i, j}$ it would result in wastage of power. So, to avoid loss we should allocate power such that the following condition holds:

$$
\gamma_{s, i->j} \geq \gamma_{s, j}+\gamma_{i, j} .
$$

After this the value of $R_{s, j}$ is given by:

$$
R_{s, j}=\log _{2}\left(1+\gamma_{s, j}+\gamma_{i, j}\right) .
$$

Our target is to distribute transmit power among NOMA CR users such that higher spectral efficiency and fairness among the communicating nodes is achieved while both SIC and interference temperature constraints are ensured. The objective consists of two parts, the goal to maximize the rate is written as $\max \left(R_{s, i}+R_{s, j}\right)$. To achieve fairness, it is required that the difference in $R_{s, i}$ and $R_{s, j}$ is minimum. These two can be combined into a single objective as $\max \left(R_{s, i}+R_{s, j}\right)-$ $\left(\left|R_{s, i}-R_{s, j}\right|\right)$. Then problem of achieving fairness in the rates of the users can be written mathematically as:
P1:

$$
\begin{aligned}
& \max \left(R_{s, i}+R_{s, j}\right)-\left(\left|R_{s, i}-R_{s, j}\right|\right) \\
& \text { s.t. } \\
& P_{s, i}+P_{s, j} \leq P_{T}, \\
& P_{i, j} \leq P r \\
& \psi \leq P_{s, j}-P_{s, i}, \\
& \left|f_{s, k}\right|^{2}\left(P_{s, i}+P_{s, j}\right) \leq I_{t h}, \\
& P_{i, j}\left|f_{i, k}\right|^{2} \leq I_{t h}, \\
& \gamma_{s, i->j} \geq \gamma_{s, j}+\gamma_{i, j} .
\end{aligned}
$$

The objective is given in (8.1), the constraint in (8.2) ensures that the transmit power of the ST is within the power budget. Similarly, (8.3) guarantees that the power used for relaying does not exceed the battery capacity where $\operatorname{Pr}$ denotes the battery capacity of the user-i. For successful SIC at the user$i$, a minimum gap is required in the received powers, this is achieved by (8.4). The value of $\psi$ is given as $\psi=\frac{\tau}{\left|h_{s, i}\right|^{2}}$, where $\tau$ represents the minimum gap requirement in the received powers. Constraints (8.5) and (8.6) protect the PR from high interference in first and second time slot, respectively. The $I_{t h}$ denotes the values of tolerable interference threshold.

\section{Proposed Scheme}

We aim to achieve fairness in the rates of the secondary users. The bisection method is a well known iterative root finding method that provides fast convergence [39]- [40]. Bisection method can be used to find the optimal value of a one dimensional differentiable function having single extremum in the given interval. In this work we employ bisection method to benefit form the fast convergence of this technique in optimization problems [39]. Fairness dictates that the rates of both users should be as close as possible, ideally, should have the same value. We can achieve this target through bisection method [40]. The steps of the proposed technique are shown in Algorithm 1. The bisection method works by adjusting

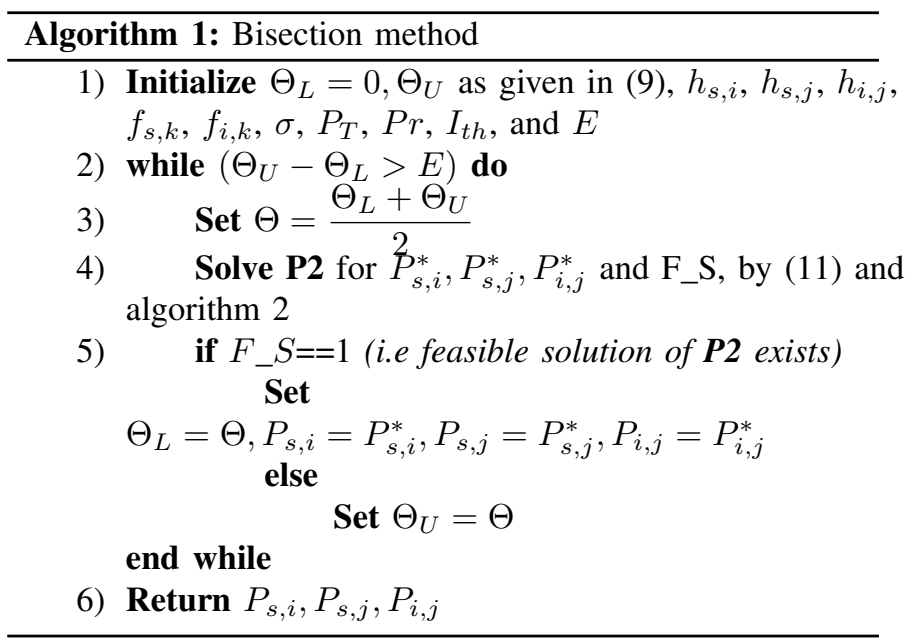

the values of the lower bound $\left(\Theta_{L}\right)$ and the upper bound $\left(\Theta_{U}\right)$ so that they converge to the optimal value $(\Theta)$. First, $\Theta_{L}$ is set to zero because the sum rate of the system can 
have a minimum value of zero. The upper bound is set to the maximum achievable rate. This is obtained when all the available power is used for the transmission of either the user$i$ or the $u s e r-j$, i.e:

$$
\begin{aligned}
\Theta_{U}= & \max \left\{\log _{2}\left(1+\frac{P_{T}\left|h_{s, i}\right|^{2}}{\sigma^{2}}\right), \frac{1}{2} \log _{2}(1+\right. \\
& \left.\left.\frac{P_{T}\left|h_{s, j}\right|^{2}}{\sigma^{2}}+\frac{P r\left|h_{i, j}\right|^{2}}{\sigma^{2}}\right)\right\},
\end{aligned}
$$

Note that in (9), the first term shows the achievable rate of user $-i$ and the second term is the rate of the IoT user- $j$. In this case, the rate of IoT user- $j$ does not suffer from interference because the ST allocates all the power for the transmission of the user- $j$. Similarly, in the first term we do not have $\frac{1}{2}$ because all the available power and time is being allocated for the transmission of user- $i$. So, the first term in (9) denotes the rate of $u s e r-i$ when all the available power and time is allocated for user- $i$, and the second term is the rate of $u s e r-j$ when all the power is allocated for the transmission of user- $j$. The $\Theta$ is calculated by averaging $\Theta_{L}$ and $\Theta_{U}$. Then, the problem (denoted as P2) is solved to get the values of $P_{s, i}, P_{s, j}, P r_{i, j}$ and F_S. F_S is a binary variable that is obtained from the algorithm 2. If $\mathrm{F} \_\mathrm{S}=1$, it means that the problem has a feasible solution otherwise the problem does not have a feasible solution. If $\mathbf{P 2}$ has a feasible solution then all the powers are updated and $\Theta_{L}$ is set equal to $\Theta$ (i.e the value of fair rate increases). Otherwise, if feasible solution does not exist the upper bound $\left(\Theta_{U}\right)$ is set equal to $\Theta$ that decreases the value of fair rate. This process is repeated until the difference between the upper and lower bound decreases below a certain tolerable error value $E$.

For a given value of fair rate $(\Theta)$, we are required to find $P_{s, i}, P_{s, j}$ and $P_{i, j}$ such that $R_{s, i} \geq \Theta, R_{s, j} \geq \Theta$ and (8.2)(8.7) are satisfied. This is a feasibility problem and is written as:

$$
\begin{aligned}
& \text { P2: } \quad \text { Find } P_{s, i}, P_{s, j}, P_{i, j}, \\
& \text { s.t. } R_{s, i} \geq \Theta, R_{s, j} \geq \Theta,(8.2),(8.3),(8.4),(8.5) \text {, } \\
& (8.6),(8.7) \text {. }
\end{aligned}
$$

At optimality, $R_{s, i}$ and $R_{s, j}$ will be as close to $\Theta$ as possible without violating any constraint. Thus, $R_{s, i} \geq \Theta$ holds with equality and the value of $P_{s, i}$ is given by:

$$
P_{s, i}^{*}=\frac{\left(2^{2 \Theta}-1\right) \sigma^{2}}{\left|h_{s, i}\right|^{2}} \text {. }
$$

In the case of $R_{s, j}=\Theta$, (8.4) might be violated. Hence, at optimality we have $R_{s, j} \geq \Theta$ i.e.:

$$
\frac{1}{2} \log _{2}\left(1+\frac{P_{s, j}\left|h_{s, j}\right|^{2}}{P_{s, i}\left|h_{s, j}\right|^{2}+\sigma^{2}}+\frac{P_{i, j}\left|h_{i, j}\right|^{2}}{\sigma^{2}}\right) \geq \Theta .
$$

For the solution, we first solve (12) for equality, in the absence of relaying. That is, we find $P_{s, j}$ such that:

$$
\frac{1}{2} \log _{2}\left(1+\frac{P_{s, j}\left|h_{s, j}\right|^{2}}{P_{s, i}^{*}\left|h_{s, j}\right|^{2}+\sigma^{2}}\right)=\Theta .
$$

For the given $P_{s, i}^{*}$, the value of $P_{s, j}$ is upper bounded as $P_{s, j} \leq P_{T}-P_{s, i}^{*}$ and $P_{s, j} \leq \frac{I_{t h}-P_{s, i}^{*}\left|f_{s, k}\right|^{2}}{\left|f_{s, k}\right|^{2}}$. Similarly, $P_{s, j}$ is lower bounded as $P_{s, j} \geq \psi+P_{s, i}^{*}$. If the value of $P_{s, j}$ violates $P_{s, j} \geq \psi+P_{s, i}^{*}$ for (13), then $P_{s, j}$ is set equal to $\psi+P_{s, i}^{*}$. Similarly if upper bound is violated, the value of $P_{s, j}$ is adjusted to satisfy all the constraints. This solution value is referred to as $P_{s, j}^{*}$. The adjustment of $P_{s, j}^{*}$ according to the bounds is shown in steps (1) and (2) of Algorithm 2. Then if at $P_{s, i}^{*}$ and $P_{s, j}^{*}$, we have $\frac{1}{2} \log _{2}\left(1+\frac{P_{s, j}^{*}\left|h_{s, j}\right|^{2}}{P_{s, i}^{*}\left|h_{s, j}\right|^{2}+\sigma^{2}}\right) \leq \Theta$, we find $P_{i, j}$ so that (12) holds with equality. This solution value is represented as $P_{i, j}^{*}$ and is given by:

$$
P_{i, j}^{*}=\left(\left(\left(2^{2 \Theta}-1\right)-\frac{P_{s, j}\left|h_{s, j}\right|^{2}}{P_{s, i}\left|h_{s, j}\right|^{2}+\sigma^{2}}\right) \frac{\sigma^{2}}{\left|h_{i, j}\right|^{2}}\right)^{+},
$$

this is the step 3 of Algorithm 2. Then $\mathbf{P 2}$ is said to have a feasible solution if at $P_{s, i}^{*}, P_{s, j}^{*}$ and $P_{i, j}^{*}$, all the constraints of $\mathbf{P 2}$ hold. The steps are summarized in Algorithm 2. In

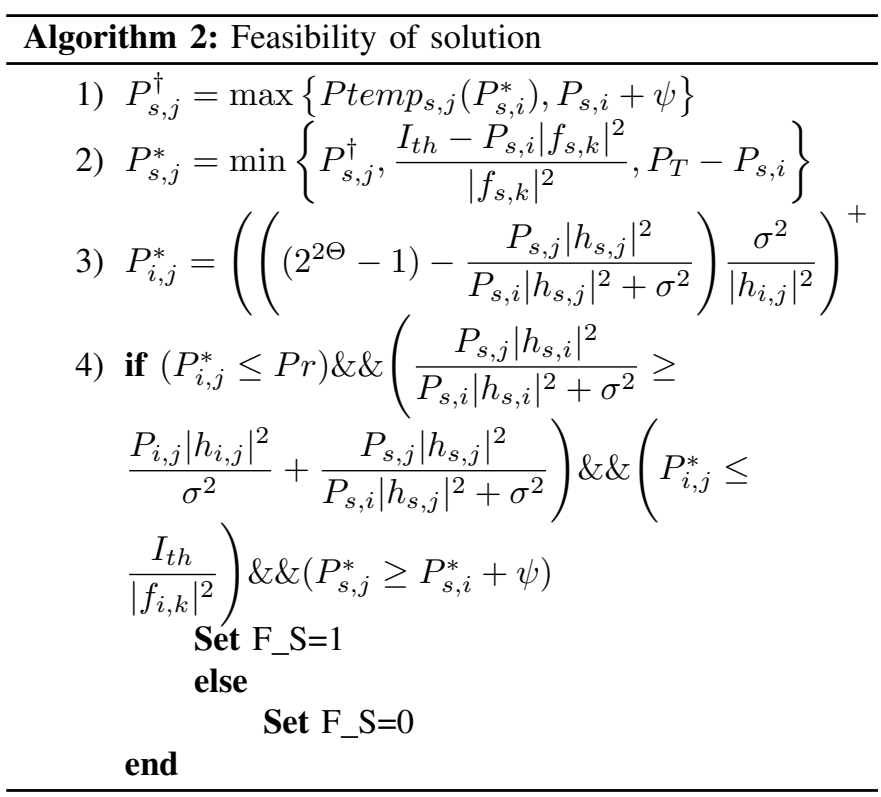

algorithm 2, Ptemp $p_{s, j}\left(P_{s, i}^{*}\right)$ is the power required by the $u$ ser $-j$ to achieve the required rate $\Theta$, without relaying and is given by:

$$
\operatorname{Ptemp}_{s, j}\left(P_{s, i}^{*}\right)=\frac{\left(2^{2 \Theta}-1\right)\left(P_{s, i}^{*}\left|h_{s, j}\right|^{2}+\sigma^{2}\right)}{\left|h_{s, j}\right|^{2}} .
$$

This gives us the solution for power allocation to achieve fairness in a NOMA based cooperative underlay IoT network.

\section{Simulation Results}

For the simulations, we consider Rayleigh fading channels and the values of $\sigma^{2}, \operatorname{Pr}, \psi$ and $E$ are taken as $0.01,1 \mathrm{~W}, 0.2 \mathrm{~W}$ and $10^{-8}$, respectively. We employ Jain's fairness index to evaluate the the proposed scheme in terms of fairness [41]. The Jain's fairness index is given as:

$$
F I=\frac{\left(R_{s, i}+R_{s, j}\right)^{2}}{2\left(R_{s, i}^{2}+R_{s, j}^{2}\right)} .
$$




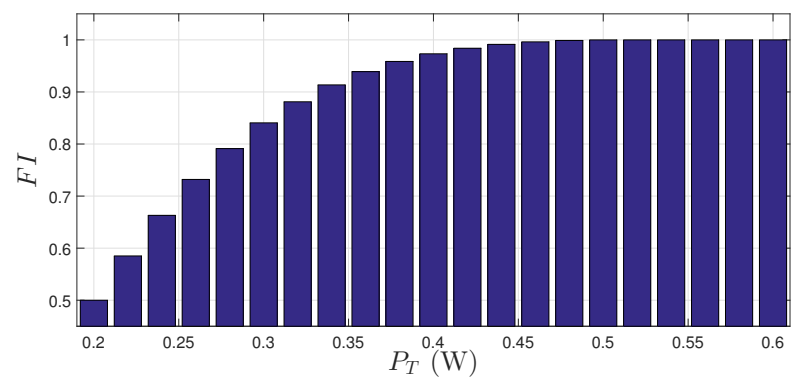

Fig. 2: Impact of the power budget of the ST $\left(P_{T}\right)$ on the FI of the system

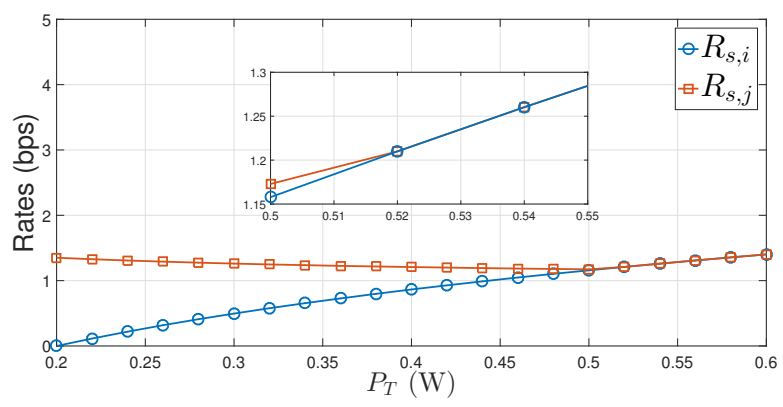

Fig. 3: The effect of increasing $P_{T}$ on the rates of the users

For $I_{t h}=1 \mathrm{~W}$, the impact of $P_{T}$ on the fairness of the system is presented in Fig. 2. Higher values of $P_{T}$ result in increased fairness of the system. Figure 3 shows that for $P_{T}<0.52$, $R_{s, i} \neq R_{s, j}$ and the value of $F I$ in this interval is less than 1 . It can be seen from Fig. 4 that $P_{i, j}=0$, for $P_{T}<0.52$. If $P_{s, j}$ is reduced to increase the fairness this will result in violating (8.4). The Fig. 4 shows that the power allocated by the ST to both the IoT users increases with $P_{T}$. However, the increase in $P_{T}$ from 0.2 to 0.5 results in increasing the rate of the user$i$ whereas the rate of the user- $j$ decreases. This is because, although the power allocated for transmission of the user$j$ has increased, the interference faced by the user has also increased. The disadvantage of high interference temperature overshadows the benefit of increasing $P_{s, j}$ and so reduction in $R_{s, j}$ is observed. On the other hand, increasing $P_{T}$ results in greater value of $R_{s, i}$ because the IoT user- $i$ does not face any interference while decoding its signal due to SIC. For $P_{T} \geq 0.52$, the rate of the user- $j$ in the first time slot is less than $R_{s, i}$. Thus, at this point the IoT user- $i$ starts to allocated power for relaying the data to the user- $j$. As a result, $R_{s, j}$ becomes equal to $R_{s, i}$ for $P_{T} \geq 0.52$. Note that, the cooperation could not play a role in fairness for $P_{T}<0.52$ because in this interval $R_{s, j}$ was greater than $R_{s, i}$. Thus, if any power was allocated for relaying it would have resulted in further decreasing the fairness. This is why for $P_{T}<0.52$ we have $P_{i, j}=0$.

The effect of $I_{t h}$ on the fairness is shown in Fig. 5. The values of $P_{T}$ was taken to be $1 \mathrm{~W}$. Generally, increasing $I_{t h}$ has a positive impact on $F I$. The Fig. 5 shows that for $I_{t h}<0.22$, the value of $F I<1$. The unfairness at the lower values of $I_{t h}$ is because the $I_{t h}$ upper bounds the transmit power of the system. Hence, at these values of $I_{t h}$ when power is

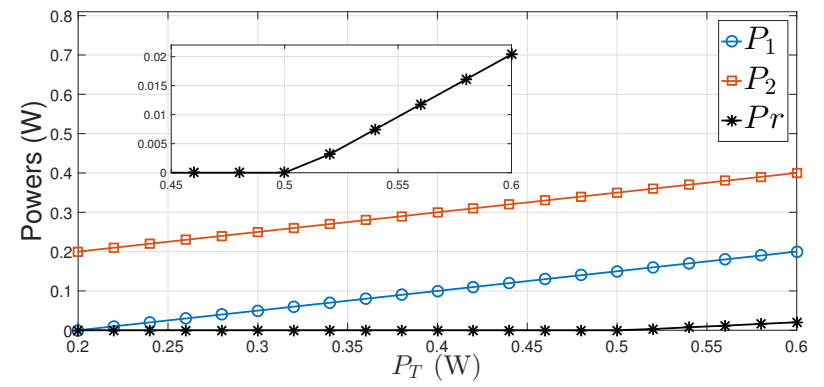

Fig. 4: Power budget of the ST $\left(P_{T}\right)$ VS the power allocated for the transmission of each user

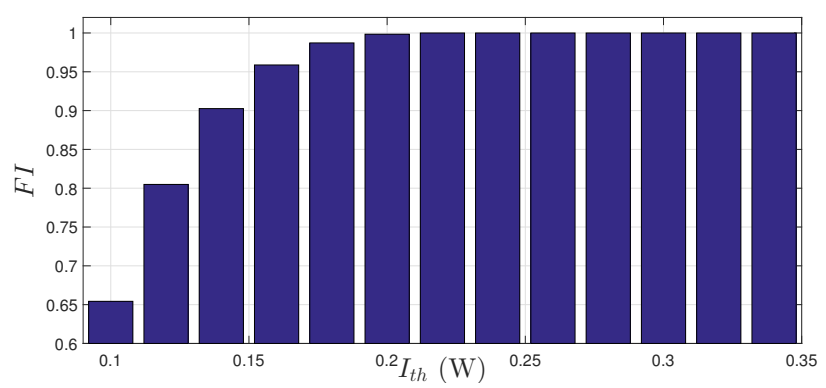

Fig. 5: Effect of increasing the interference threshold $\left(I_{t h}\right)$ on the fairness index $(\mathrm{FI})$

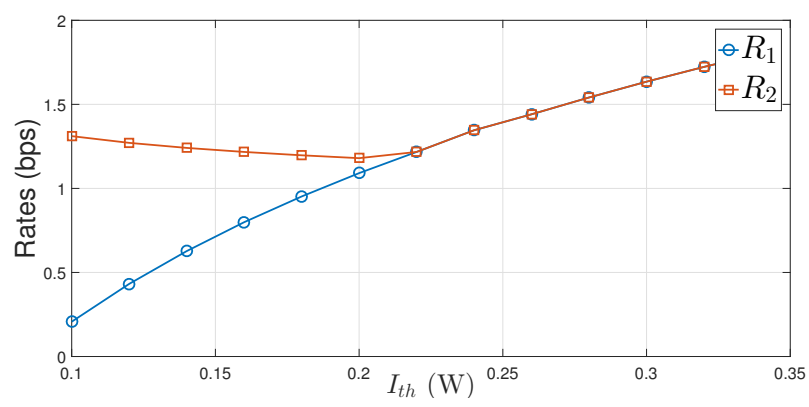

Fig. 6: Impact of interference threshold $\left(I_{t h}\right)$ on the rates of the users

allocated to satisfy (8.4) it results in $R_{s, j}>R_{s, i}$. Increasing the threshold from 0.22 results in $R_{s, i}=R_{s, j}$ as shown in Fig. 6. Increasing $I_{t h}$ for $I_{t h}<0.22$ results in decreasing $R_{s, j}$ because of the increase in interference temperature as discussed before. At $I_{t h}=0.22$ the direct transmission rate of the user- $j$ is less compared to the rate of the user- $i$. So, the user- $i$ invests power for relaying the data to the user- $j$. This results in enhanced fairness at $I_{t h}=0.22$. After this point, an increase in the values of $I_{t h}$ does not affect the fairness of the system.

The impact of $P_{T}, I_{t h}$ and $\operatorname{Pr}$ on the system's achievable rate is presented in Fig. 7 and Fig. 8. The Fig. 7 compares the effect of $P_{T}$ on the sum rate for different values of $I_{t h}$. It is clear that for any value of $I_{t h}$ the sum rate increases with $P_{T}$, initially. However, after a point, an increase in $P_{T}$ has no effect on the rate. This is because at this point the interference has become equal to the $I_{t h}$. Thus, if any additional power is allocated for the transmission this would result in violating the interference threshold. Larger values of 


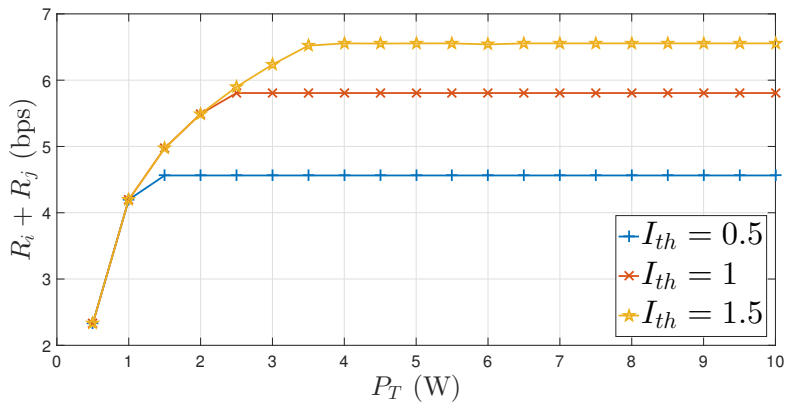

Fig. 7: Sum rate of the system VS the power budget of the ST $\left(P_{T}\right)$ with different $I_{t h}$

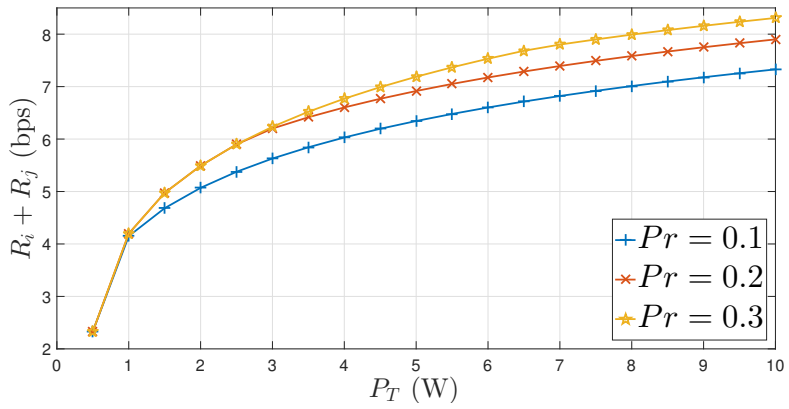

Fig. 8: Impact of power budget of the ST on the sum rate of the system for different values of the battery capacity of the user-iuser-i

$I_{t h}$ allows transmission with more power. Hence, the system offers higher sum rate for greater values of $I_{t h}$. Figure 8 presents the impact of $P_{T}$ on the rate for different values of $P r$. In this case, we have considered $I_{t h}=2$, for making the effect of $\operatorname{Pr}$ more prominent. At $P_{T} \leq 1$, the rate for all cases is same. This is because at small values of $P_{T}$ the decoding rate of user-j's data at the user- $i$ is small. If more power is allocated for relaying it would result in violating (8.7). Hence, at this point same relaying power is used in all three cases. However, with an increase in $P_{T}$ the decoding rate of the user$j$ 's data also increases and so the systems with greater values of $\operatorname{Pr}$ can invest more power for relaying. Thus, at these points larger $\mathrm{Pr}$ results in greater value of sum rate.

\section{CONCLUSION}

In this paper, we considered the problem of achieving fairness among the IoT users in a cooperative NOMA CR system. The constraints of interference threshold, power limit, relaying-decoding rate and SIC gap requirement were taken into consideration. We proposed a bisection method based solution for power allocation. The simulations proved the efficiency of the proposed scheme. It was made clear by the results that perfect fairness is not achievable at small values of power budget and interference threshold. However, the proposed technique provides promising results for small power budget and the fairness of the system increases rapidly with an increase in the available power and interference threshold. In the future, we will focus on achieving fairness under imperfect SIC considerations for a multiuser scenario.

\section{REFERENCES}

[1] W. U. Khan, Z. Ali, M. Waqas, G. A. S. Sidhu, Efficient power allocation with individual qos guarantees in future small-cell networks, AEUInternational Journal of Electronics and Communications 105 (2019) $36-41$.

[2] P. E. Omiyi, M. M. Nasralla, I. U. Rehman, N. Khan, M. G. Martini, An intelligent fuzzy logic-based content and channel aware downlink scheduler for scalable video over ofdma wireless systems, Electronics 9 (7) (2020) 1071.

[3] M. M. Nasralla, A hybrid downlink scheduling approach for multi-traffic classes in lte wireless systems, IEEE Access 8 (2020) 82173-82186.

[4] L. Xu, X. Yu, A. Gulliver, Intelligent outage probability prediction for mobile iot networks based on an igwo-elman neural network, IEEE Transactions on Vehicular Technology (2021).

[5] L. Xu, H. Wang, T. A. Gulliver, Outage probability performance analysis and prediction for mobile iov networks based on ics-bp neural network, IEEE Internet of Things Journal (2020).

[6] A. S. Marcano, H. L. Christiansen, Performance of non-orthogonal multiple access (noma) in mmwave wireless communications for $5 \mathrm{~g}$ networks (2017) 969-974.

[7] W. U. Khan, F. Jameel, M. A. Jamshed, H. Pervaiz, S. Khan, J. Liu, Efficient power allocation for noma-enabled iot networks in $6 \mathrm{~g}$ era, Physical Communication 39 (2020) 101043.

[8] F. Fang, J. Cheng, Z. Ding, Joint energy efficient subchannel and power optimization for a downlink noma heterogeneous network, IEEE Transactions on Vehicular Technology 68 (2) (2018) 1351-1364.

[9] W. U. Khan, J. Liu, F. Jameel, V. Sharma, R. Jantti, Z. Han, Spectral efficiency optimization for next generation noma-enabled iot networks, IEEE Transactions on Vehicular Technology (2020).

[10] M. Liu, T. Song, J. Hu, J. Yang, G. Gui, Deep learning-inspired message passing algorithm for efficient resource allocation in cognitive radio networks, IEEE Transactions on Vehicular Technology 68 (1) (2018) 641-653.

[11] M. Abbasi, H. Rezaei, V. G. Menon, L. Qi, M. R. Khosravi, Enhancing the performance of flow classification in sdn-based intelligent vehicular networks, IEEE Transactions on Intelligent Transportation Systems (2020).

[12] S. Jacob, V. G. Menon, P. Shynu, F. S. KS, B. Mahapatra, S. Joseph, Bidirectional multi-tier cognitive swarm drone $5 \mathrm{~g}$ network, in: IEEE INFOCOM 2020-IEEE Conference on Computer Communications Workshops (INFOCOM WKSHPS), IEEE, 2020, pp. 1219-1224.

[13] X. Pei, H. Yu, Y. Chen, M. Wen, G. Chen, Hybrid multicast/unicast design in noma-based vehicular caching system, IEEE Transactions on Vehicular Technology (2020).

[14] Z. Wang, M. Wen, S. Dang, L. Yu, Y. Wang, Trajectory design and resource allocation for uav energy minimization in a rotary-wing uavenabled wpcn, Alexandria Engineering Journal 60 (1) (2020) 1787-1796.

[15] W. U. Khan, Z. Yu, S. Yu, G. A. S. Sidhu, J. Liu, Efficient power allocation in downlink multi-cell multi-user noma networks, IET Communications 13 (4) (2018) 396-402.

[16] W. U. Khan, F. Jameel, T. Ristaniemi, S. Khan, G. A. S. Sidhu, J. Liu, Joint spectral and energy efficiency optimization for downlink noma networks, IEEE Transactions on Cognitive Communications and Networking (2019).

[17] Y. Feng, S. Yan, Z. Yang, N. Yang, J. Yuan, Beamforming design and power allocation for secure transmission with noma, IEEE Transactions on Wireless Communications 18 (5) (2019) 2639-2651.

[18] Z. Dong, T. Shang, Q. Li, T. Tang, Adaptive power allocation scheme for mobile noma visible light communication system, Electronics 8 (4) (2019) 381.

[19] Z. Ali, G. A. S. Sidhu, M. Waqas, F. Gao, On fair power optimization in nonorthogonal multiple access multiuser networks, Transactions on Emerging Telecommunications Technologies 29 (12) (2018) e3540.

[20] F. Zhou, N. C. Beaulieu, Z. Li, J. Si, P. Qi, Energy-efficient optimal power allocation for fading cognitive radio channels: Ergodic capacity, outage capacity, and minimum-rate capacity, IEEE Transactions on Wireless Communications 15 (4) (2015) 2741-2755.

[21] T. X. Quach, H. Tran, E. Uhlemann, G. Kaddoum, Q. A. Tran, Power allocation policy and performance analysis of secure and reliable communication in cognitive radio networks, Wireless Networks 25 (4) (2019) 1477-1489.

[22] W.-C. Pao, Y.-F. Chen, S.-Y. Chuang, Efficient power allocation schemes for ofdm-based cognitive radio systems, AEU-International Journal of Electronics and Communications 65 (12) (2011) 1054-1060. 
[23] Z. Ali, G. A. S. Sidhu, M. Waqas, F. Gao, S. Jin, Achieving energy fairness in multiuser uplink cr transmission, in: 2016 IEEE Wireless Communications and Networking Conference, IEEE, 2016, pp. 1-6.

[24] W. U. Khan, F. Jameel, N. Kumar, R. Jntti, M. Guizani, Backscatterenabled efficient V2X communication with non-orthogonal multiple access, IEEE Transactions on Vehicular Technology 70 (2) (2021) 1724 1735. doi:10.1109/TVT.2021.3056220.

[25] F. Jameel, S. Zeb, W. U. Khan, S. A. Hassan, Z. Chang, J. Liu, NOMA-enabled backscatter communications: Toward battery-free IoT networks, IEEE Internet of Things Magazine 3 (4) (2020) 95-101. doi:10.1109/IOTM.0001.2000055.

[26] W. U. Khan, X. Li, M. Zeng, O. A. Dobre, Backscatter-enabled NOMA for future $6 \mathrm{G}$ systems: A new optimization framework under imperfect SIC, IEEE Communications Letters (2021) 11doi:10.1109/LCOMM.2021.3052936.

[27] Z. Ali, G. A. S. Sidhu, F. Gao, J. Jiang, X. Wang, Deep learning based power optimizing for noma based relay aided $\mathrm{d} 2 \mathrm{~d}$ transmissions, IEEE Transactions on Cognitive Communications and Networking (2021) 11doi:10.1109/TCCN.2021.3049475.

[28] Z. Ding, Y. Liu, J. Choi, Q. Sun, M. Elkashlan, I. Chih-Lin, H. V. Poor, Application of non-orthogonal multiple access in lte and $5 \mathrm{~g}$ networks, IEEE Communications Magazine 55 (2) (2017) 185-191.

[29] Z. Wei, X. Zhu, S. Sun, J. Wang, L. Hanzo, Energy-efficient fullduplex cooperative nonorthogonal multiple access, IEEE Transactions on Vehicular Technology 67 (10) (2018) 10123-10128.

[30] H. Yu, W. Duan, G. Zhang, Y. Ji, X. Zhu, J. Choi, A near optimal power allocation scheme for cooperative relay networking with noma, China Communications 16 (3) (2019) 122-131.

[31] Y. Xu, C. Shen, Z. Ding, X. Sun, S. Yan, G. Zhu, Z. Zhong, Joint beamforming and power-splitting control in downlink cooperative swipt noma systems, IEEE Transactions on Signal Processing 65 (18) (2017) 4874-4886.

[32] R. Bordón, S. M. Sánchez, S. B. Mafra, R. D. Souza, J. L. Rebelatto, E. M. G. Fernandez, Energy efficient power allocation schemes for a two-user network-coded cooperative cognitive radio network, IEEE Transactions on Signal Processing 64 (7) (2015) 1654-1667.

[33] T. Yang, H. Feng, C. Yang, R. Deng, G. Guo, T. Li, Resource allocation in cooperative cognitive radio networks towards secure communications for maritime big data systems, Peer-to-Peer Networking and Applications 11 (2) (2018) 265-276.

[34] M. Zeng, G. I. Tsiropoulos, O. A. Dobre, M. H. Ahmed, Power allocation for cognitive radio networks employing non-orthogonal multiple access, in: 2016 IEEE Global Communications Conference (GLOBECOM), IEEE, 2016, pp. 1-5.

[35] N. Zabetian, M. Baghani, A. Mohammadi, Rate optimization in noma cognitive radio networks, in: 2016 8th International Symposium on Telecommunications (IST), IEEE, 2016, pp. 62-65.

[36] W. U. Khan, et al., NOMA-enabled optimization framework for nextgeneration small-cell IoV networks under imperfect SIC decoding, IEEE Transactions on Intelligent Transportation Systems (2021) 1-1.

[37] S. Yu, et al., Optimal power allocation for NOMA-enabled D2D communication with imperfect SIC decoding, Physical Communication (2021) 101296.

[38] W. U. Khan, F. Jameel, G. A. S. Sidhu, M. Ahmed, X. Li, R. Jäntti, Multiobjective optimization of uplink NOMA-enabled Vehicleto-Infrastructure communication, IEEE Access 8 (2020) 84467-84478.

[39] M. Bartholomew-Biggs, Nonlinear optimization with engineering applications, Vol. 19, Springer Science \& Business Media, 2008.

[40] R. Burden, J. Faires, The bisection method, Numerical Analysis (2011) 48-56.

[41] R. Jain, D.-M. Chiu, W. Hawe, A quantitative measure of fairness and discrimination for resource allocation in shared computer systems, 1984, Cited on (1984) 33. 